\title{
The Mimicry Among Us: Intra- and Inter-Personal Mechanisms of Spontaneous Mimicry
}

\author{
Andrew J. Arnold ${ }^{1} \cdot$ Piotr Winkielman ${ }^{1,2}$ i
}

Published online: 13 November 2019

(c) The Author(s) 2019

\begin{abstract}
This review explores spontaneous mimicry in the context of three questions. The first question concerns the role of spontaneous mimicry in processing conceptual information. The second question concerns the debate whether spontaneous mimicry is driven by simple associative processes or reflects higher-order processes such as goals, intentions, and social context. The third question addresses the implications of these debates for understanding atypical individuals and states. We review relevant literature and argue for a dynamic, context-sensitive role of spontaneous mimicry in social cognition and behavior. We highlight how the modulation of mimicry is often adaptive but also point out some cases of maladaptive modulations that impair an individuals' engagement in social life.
\end{abstract}

Keywords Mimicry $\cdot$ Social context · Imitation · Social cognition · Mirroring · Embodiment $\cdot$ Emotion concepts $\cdot$ Emotion understanding $\cdot$ Contagion

\section{Introduction}

In order to characterize processes that allow individuals to understand, coordinate with, and influence others, much recent research has focused on intentional and spontaneous imitation. Intentional imitation occurs when an individual deliberately replicates the actions of another. Such imitation contributes to joint action and social learning-the transmission of skills, norms, traditions, and rituals. It is also important in social communication, such as signaling the desire for connection (e.g., clapping together), indicating group membership (e.g., dressing alike), liking, but also derision (e.g., parodying and parroting). Note that intentionally imitative behaviors are often complex, reflecting the mimic's considerations about the goals and usefulness of observed and initiated actions (Csibra and Gergely 2009; Mesoudi 2009; Tomasello et al. 1993). Though our review concerns spontaneous imitation, several insights were inspired by the above literature.

Piotr Winkielman

pwinkiel@ucsd.edu

1 Department of Psychology, University of California, San Diego, 9500 Gilman Drive, La Jolla, CA 92093-0109, USA

2 SWPS University of Social Sciences and Humanities, Warsaw, Poland 
Here, our focus is on a more rudimentary (but not necessarily simpler) form of imitation: spontaneous mimicry - a phenomenon where merely observing another individual's behavior implicitly elicits a corresponding action in the observer, without an explicit instruction or intention to do so. Mimicry appears to have phylogenetic roots and occurs in non-human primates (e.g., contagious yawning and smiling; Anderson et al. 2004; Palagi et al. under review). Such uninstructed mimicry occurs across a variety of modalities and effectors (e.g., face, voice, body, etc.). Once a stimulus appears, spontaneous mimicry can arise anywhere from fractions of a second (e.g., finger mimicry; Leighton et al. 2010), around a half-second (e.g., facial mimicry; Dimberg 1982), to several seconds (e.g., yawning, pronunciation, and postural mimicry; Tiedens and Fragale 2003).

In this review, we address three central questions-all subject to lively debates in the literature. The first question is quite broad and concerns the role of spontaneous mimicry in processing higher-order, conceptual information. The second question asks whether spontaneous mimicry is "rigid"-reflecting simple motor reproduction, or "elastic"-reflecting context, from lower-level aspects like similarity, emotional state, to higher-order goals and intentions. The third question addresses the implications of these debates for understanding individuals with atypical traits or states. With these questions in mind, we structure our review as follows: We begin by introducing the framework of embodiment, as it helps to locate research on spontaneous mimicry within the larger theoretical context. Then, we review how mimicry occurs during stimulus perception, outline the role of sensorimotor feedback in the processing of emotional information, and consider top-down modulation of mimicry phenomena. Following that, we discuss spontaneous mimicry in the context of important social variables, including power and cooperation. We also discuss work suggesting sensitivity of mimicry to more rudimentary variables like agent-perceiver similarity. Finally, we discuss the implications of all this work for investigations of atypical individuals and atypical states, including individuals with autism spectrum conditions and those experiencing loneliness. This allows us to highlight how mimicry is modulated by reward processes and variables related to perception and categorization of bodily states, such as alexithymia and interoception.

Our general perspective in this review is compatible with recent reviews that emphasize the modulation of mimicry by social context (Duffy and Chartrand 2015; Fischer and Hess 2017; Hess and Fischer 2013). However, we put more emphasis on how such modulation reflects an interaction of basic and higher-order cognitive and affective processes, and elaborate on specific mechanisms. Furthermore, we also emphasize the cognitive functions of imitation and link spontaneous mimicry to basic mechanisms of emotion memory, emotion recognition, and emotion understanding. Finally, we also focus on the modulatory role of perceptual similarity and non-cognitive variables, such as reward processing and bodily sensitivity, and its atypical operation in autistic and lonely individuals. As such, our perspective highlights that whereas some cases of mimicry modulation are driven by higher-order cognitive and social goals, other cases are better understood as reflecting perceptual and affective processes, potentially leading to non-optimal or even maladaptive mimicry modulation.

\section{Mimicry and Embodiment}

Before going into more specific mimicry phenomena, it helps to situate research on spontaneous mimicry within broader notions about the relationship between perception, cognition, and action. One theoretical approach that guides much of our thinking is the framework of embodied cognition. In brief, embodiment theories propose that higher-level 
processing is grounded in the organism's sensory and motor experiences. Accordingly, such frameworks are often called grounded cognition theories (Barsalou 1999; Clark 1999; Wilson 2002). Such theories propose that processing of information (e.g., about objects, tools, faces, gestures, personality traits, and even abstract social, moral, emotional, or motivational concepts), draws on perceptual, somatosensory, and motor resources. Such resources are engaged during the actual encounter with the stimulus, and when thinking about the stimulus "off-line" (in its absence). For example, when perceivers process a facial expression or a bodily gesture, they could draw on their own facial or arm muscles or central sensory-motor circuits. Similarly, when thinking about whether some concept (e.g., an orange) includes some property (e.g., sweet), the perceiver could partially recreate the sensory experience (e.g., tasting the orange). This recreation could involve activation of peripheral resources (e.g., salivation), or central resources (e.g., gustatory cortex). Critically, such a reenactment - called embodied simulation — does not require any peripheral or behavioral manifestations, as it presumably can run solely on the brain's central modality systems, and only needs to re-instantiate enough of the original experience to be useful in further processing. Further, as we will see, the nature of situational demands and relative stimulus/concept ambiguity shape this process. In any case, as a result, conceptual processing could be "influenced", "informed", "associated", "dependent", and perhaps even "constituted" by such embodied simulations.

The embodiment framework has long been used to better understand processing of social and emotional information (Niedenthal et al. 2005; Schubert and Semin 2009; Winkielman et al. 2015). However, only recently, researchers have started to emphasize the full complexity of the underlying process. The latest formulation of our perspective is the CODES model-standing for context-dependent embodied simulation (Winkielman et al. 2018). As the name suggests, this model highlights that context directs the degree and impact of embodied simulation - a point especially relevant for understanding processing of emotion stimuli. CODES has several assumptions, but for the current purposes we emphasize two: (i) context shapes the extent and pattern of somatosensory and motor involvement, (ii) embodied simulation is more likely when inferential elaboration is needed (e.g., for unclear or ambiguous stimuli). The CODES model contrasts with a model of mimicry as primarily reflecting a process of context-independent, reflexive association between perception and action, where motor activity has an epiphenomenal status. The CODES model also contrasts with models of mimicry that view similarities in target-perceiver motor activity as a result of an earlier induction of a similar emotional state (e.g., seeing a target smile makes a perceiver happy which makes her smile). As we will see shortly, the assumptions of CODES are reasonably supported by empirical research. This is especially true for research on spontaneous mimicry in processing of emotion concepts and then we address its role in processing of faces and gestures. However, in the later parts of the paper, we will also cover research on atypical mimicry, emphasizing the role of additional variables (reward sensitivity, interoception) that are not captured by the CODES framework.

\section{Emotion Concepts in Facial Emotion Processing}

One key point of the embodiment framework is that spontaneous mimicry links sensorimotor representations with concepts-higher-order, more abstract mental categories. One illustrative study tested how encouraging individuals to think about emotion concepts (e.g., happiness, anger) changes their facial reactions to others' expressions and their memory 
for these expressions (Halberstadt et al. 2009). Participants were first asked to view faces of several different individuals with ambiguous expressions (50\% morph between happy and angry). For each face, participants were told to consider why each of these individuals might feel "happy" or "angry." These emotion concepts were randomly paired with the ambiguous faces. During this task, we monitored facial responses using facial electromyography (fEMG) to detect activity in the zygomaticus major ("smiling muscle") and the corrugator supercilli ("frowning muscle"). Analyses of this fEMG activity showed that the ambiguous faces paired with the "happy" concept elicited more smiling than faces paired with the "angry" concept. Thus, higher-order information about "happiness" or "anger" shaped participants' spontaneous facial responses-a pattern we also observed in other research (e.g., Niedenthal et al. 2009). Critically, in the second phase of the experiment, participants were asked to identify the exact physical expression shown earlier by each individual. To do this, they were shown a movie presenting a continuous morph between happy and angry expressions and needed to indicate which exact frame showed the previously viewed face (a correct response would be frame 50-exactly halfway). Results showed that participants' perceptual memory of the expression was biased in the direction of the previously paired concept (e.g., they remembered a face as happier when it was earlier associated with a "happy" label). Also during this second phase (counterbalanced order), participants were also asked to passively view the ambiguous 50-50 faces. The fEMG data showed that participants spontaneously smiled more to "happy" faces than "angry" faces, as if the faces were actually different. Importantly, the perceptual memory bias effect was correlated with spontaneous fEMG response during mere viewing. In sum, the results demonstrate the interplay of top-down information (emotion concepts) with a motor response (facial muscles) to encode memory of a target person. This interplay led to a shift in perceptual memory and a change in facial activity upon later viewing of the ambiguous faces. In conclusion, mimicry could be said to represent a bridge between the sensorimotor and cognitive system. With that general insight in mind, let's now look in more detail at processing of facial and gestural stimuli.

\section{Spontaneous Mimicry and Processing of Facial Emotion}

As discussed, embodied accounts propose that somatosensory and motor representations play a role in emotional processing (Barsalou 1999; Damasio 1999; Niedenthal et al. 2005; Wood et al. 2016). Consistent with this proposal, studies using functional magnetic resonance imaging (fMRI) show that merely observing facial expressions increases neural activity in somatosensory areas that represent one's own face (e.g., Keysers et al. 2010; Molenberghs et al. 2012). Further, fEMG studies show mere observation of a facial expression often leads to generation of actual facial movement(s), which tend to match the perceived facial configuration-spontaneous facial mimicry (Dimberg 1982; Lundqvist and Dimberg 1995). Thus (usually) when people see smiles, they produce incipient smiles, and when they see frowns, they produce incipient frowns. We will describe modulation of this simple matching pattern shortly, but under "default" conditions, such facial mimicry appears relatively automatic and straightforward. It also occurs very quickly-within a half- to full-second after seeing an expression (e.g., Dimberg 1982). They occur even when participants are asked to inhibit a facial response (Korb et al. 2010) or when they are asked to respond to the perceived facial stimulus in the opposite way ("counter-mimicry"; Dimberg et al. 2002). Further, spontaneous facial mimicry occurs after minimal stimulus 
input, even upon sub-threshold presentations for expressions of joy or anger (Dimberg et al. 2000). Importantly, a match between the target and subject's expression can reflect mere motor similarity but also result from underlying match in valence (via emotion induction) — suggesting that both purely imitative and evaluative processes play a role (for more, see Moody et al. 2007; Neumann et al. 2014).

Given that stimuli leading to facial mimicry can be implicitly perceived, it is possible that the mimicry itself may be implicit—not available to conscious awareness. We approached this question in a study where participants were briefly flashed happy, neutral, or angry faces and asked to identify their valence (Bornemann et al. 2012). We monitored participants' smiling and frowning activity using fEMG. We found that happy and angry faces produced matching, congruent patterns of fEMG activity (smile to a smile, frown to a frown). But, were these mimicry reactions consciously available? To test this, we asked one group of participants to do the valence detection task while focusing on their feelings, including "subtle twitches" in their faces. Another group was instructed to use a visual focus strategy. The last group received no strategy instructions. Our results revealed no benefit of the feeling-focused strategy on valence detection rates, suggesting that responses to facial stimuli were not consciously available.

Most of the above results simply show correlation between perception and action. As such, they could reflect frequent associative pairing of perception and action and play no causal role in understanding of emotion or gestures. For example, when we see a smile, we usually smile, so it is not surprising that upon seeing a smile, there is a quick, spontaneous activation of congruent motor processes. Similarly, demonstrations that seeing a gesture (e.g., hand movement) is correlated with central and peripheral activation of motor processes say little about the causal role of such motor activation in gesture understanding (Cook et al. 2014a). However, some research provides evidence for the causal role of such spontaneous mimicry in emotion recognition. Preventing participants from engaging expression-relevant facial muscles, for example, sometimes impairs their ability to detect briefly presented, subtle, or ambiguous facial expressions that involve that specific muscle (Bulnes et al. 2019; Davis et al. 2017; Niedenthal et al. 2001; Oberman et al. 2007; Stel and Knippenberg 2008). In addition, studies show that inhibition of smiles results in poorer differentiation between "true" and "false" smiles - a process that relies on processing of subtle facial distinctions (Maringer et al. 2011; Rychlowska et al. 2014). Finally, some studies show that natural lesions and experimental (temporary) inactivation of sensory-motor areas can impair emotion recognition (Adolphs et al. 2000; Pitcher et al. 2008).

Having said that, it is clear that more work needs to be done to fully understand the boundary conditions for the role of facial mimicry in emotion recognition. In fact, we are far from proposing that mimicry is always involved in the processing of facial expressions (or that it always necessary). For instance, in some studies observers mimicked emotional faces, but the degree of mimicry was not correlated with decoding accuracy (Blairy et al. 1999). However, when the task is more conceptually complex, as in perceiving authenticity of a smile, fEMG activity did predict judgments (Korb et al. 2014).

Further, Calder et al. (2000) found that three patients with Mobius syndrome (a congenital condition that causes facial paralysis, thus preventing mimicry) correctly categorized emotional faces, with impairments only at high levels of recognition difficulty. Another study found that individuals with Mobius syndrome do not differ in facial emotion-recognition accuracy compared to controls (Rives-Bogart and Matsumoto 2010), though such individuals also had a life to learn alternative recognition strategies. Similarly, as discussed more fully below, autistic participants who show atypical mimicry may also develop alternative routes to recognition. Here, the critical point is that typical perceivers may activate 
sensorimotor networks, when appropriate, in the course of everyday processing. Such activations can be useful for recognition, especially when the recognition cannot be achieved via a highly-automated pattern-recognition strategy. To be clear, motor activation is probably not needed to recognize a large teeth-bearing smile, but could be quite useful in subtler recognition with subtle, ambiguous, or brief expressions (i.e., much of real social life).

\section{Modulation of Spontaneous Mimicry by Social Context}

Sometimes, spontaneous mimicry follows simple, direct-matching rules (e.g., yawnto-yawn, smile-to-smile, finger-to-finger, etc.). However, it is also clear that individuals' imitative behaviors are influenced by the social context in which the interaction occurs. Spontaneous mimicry is sensitive to interpersonal cues such as prosociality (Leighton et al. 2010), group membership (Bourgeois and Hess 2008), attitudes (Likowski et al. 2008), competition (Weyers et al. 2009), empathy level (Sonnby-Borgstrom 2016), and desire for affiliation (for theoretical elaboration, see Kavanagh and Winkielman 2016). As a result, recent theories of facial mimicry suggest that it is substantially dependent on higher-level processes such as goals, appraisals, and meaning-making (Fischer and Hess 2017; Hess and Fischer 2013). Importantly, as we emphasize later, the broader notion of "context" comprises higher-order cognitive variables, lower-level perceptual variables, as well as individual states and traits that modulate the presence and intensity of spontaneous facial mimicry (for a review, see Seibt et al. 2015).

\section{Power in Context}

One stark demonstration of flexibility in mimicry in social context comes from research on power-the subjective sense of control and authority in an interaction. Carr et al. (2014) found that spontaneous fEMG responses to target's faces jointly depend on the participants' own power level, the status level of the target, and the emotion of the target's expression. In this study, all perceivers (regardless of their own power state) responded with a quick (under $1 \mathrm{~s}$ ) increase in corrugator activation (frowning) to angry high-status targets, compared to low-status targets. Interestingly, low-power perceivers also showed zygomaticus activation (smiling) in response to all target expressions (joy and anger). Those zygomaticus responses were slower (2-4 s) than corrugator, though-creating an interesting pattern of quick mimicry (matching) of anger and somewhat later counter mimicry (smiling to anger). Also interestingly, high-power perceivers only exhibited standard smile mimicry toward low-status targets but did not mimic the smiles of high-status targets. Instead, highpower perceivers smiled more only when high-status targets expressed anger, potentially reflecting elements of schadenfreude, competitive derision, or perhaps even appeasement.

Though assigning a specific interpretation to these facial response patterns is hard, they clearly demonstrate that spontaneous facial responses fundamentally change according to contextual cues of social hierarchy. As such, these results show a limited explanatory scope of direct-matching theories that would predict a straightforward correspondence between perceiver and target expression. Instead, these results suggest that spontaneous facial mimicry can be socially driven, whereby interaction goals, emotional appraisals, and situational constraints all converge in constructing the perceiver's "appropriate" facial response (Hess and Fischer 2013). 
While differential levels of power within a dyad can modulate spontaneous facial mimicry to emotional expressions, does this influence extend to more rudimentary (simpler, less emotional) forms of mimicry? This question was addressed by a series of studies that manipulated social status or power levels in the participant and tested its effect on a basic task that assesses automatic imitation of observed finger movements (Farmer et al. 2016). Results revealed that status and power manipulations did not impact spontaneous finger mimicry in this task. However, it is important to note that (a) the target finger did not reflect any specific social identity (or power differential, as in the above study), and (b) the finger movements themselves had no distinct social intent or meaning. This latter point was addressed by a recent study that examined mimicry of hand movements with clear social intent - an antisocial middle finger or prosocial thumbs up (Cracco et al. 2018). Results showed that mimicry was stronger for prosocial, compared to antisocial, gestures. In addition, priming a prosocial context increased this difference (i.e., when it is even less acceptable to produce a middle finger). Therefore, again, it appears that the intention of a given behavior (facial expression or gesture) as well as the context in which it occurs combine to allow the meaning of the behavior to shape the process of spontaneous mimicry-a point that will be elaborated next.

\section{Robots in Context}

The discussion above highlights the sensitivity of mimicry to contextual influences. This message is reinforced and extended by work from our lab using robots-in their mechanical and humanoid forms. Using such robots sheds light on mechanisms of mimicry that are specific to human interactions or represent general properties of our mental system. As we discuss below, some features and contexts strongly influence mimicry. However, mimicry is also quite robust to variations of other features. Furthermore, whereas some modifying variables operate on a high, conceptual level, others operate on more basic, perceptual levels.

One question about mimicry that is particularly suitable to exploration with androids is the role of agent-perceiver similarity. Most mimicry theories assume that spontaneous imitation requires that the observed agent is somehow "similar" to the perceiver. Yet "similarity" is a complex concept, where the relevant dimensions vary depending on context. Specifically, research highlights that mimicry can be influenced by: (a) visual similarity (Nadel et al. 2006; Press et al. 2005; Weyers et al. 2006), (b) similarity in type or biological nature of motion (Calvo-Merino et al. 2006; Chaminade et al. 2005), and (c) psychological similarity, including relatability, liking, and intentionality (Bourgeois and Hess 2008; Goldman and Sripada 2005; Likowski et al. 2008). In order to examine these different components, we conducted a series of studies using human and robot targets. Some of these experiments focused on gestures, while others focused on facial expressions.

\section{Gestures}

To explore the role of similarity in outward appearance and similarity in motion, we used a robot (Repliee Q2) that is based on an actual human (Hofree et al. 2015). Participants watched videos of this robot in its mechanical appearance (and mechanical motion), in its android appearance (human-like appearance and mechanical motion), and videos of a matched human agent (human appearance and human motion). When passively observing the robot's arm actions, participants spontaneously mimicked it, as evidenced by EMG 
from their arm. This shows that spontaneous mimicry can occurs in response to a nonintentional agent, even without human-like appearance. More importantly, participants mimicked the human with greater intensity than the robot, even when it appeared in its android, human-like appearance. This shows that even in mere observation, the similarity of the viewed agent's motion (not its outward appearance) plays an important role.

\section{Facial Emotion}

To further understand the features controlling spontaneous mimicry, we examined spontaneous responses to expressions of anger and joy generated by a human and an android (Hofree et al. 2014). The human and android expressions were very similar in appearance and temporal dynamics (for details see Wu et al. 2009). As expected, participants spontaneously mimicked the human control. They also spontaneously mimicked the android, with remarkable fidelity in timing and intensity of the response. However, we also discovered two additional things. First, participants' mimicry of androids depended on their ratings of human-likeness in terms of physical similarity. Surprisingly, ratings of psychological similarity to humans did not influence mimicry (measured using IDAQ, Waytz et al. 2010). Second, participants mimicked the android even when they felt uncomfortable with it and described it as "creepy". In sum, these studies again suggest that physical similarity (not psychological similarity or emotional comfort) plays an important role in spontaneous mimicry, at least of androids. This is different from human-human mimicry where psychological similarity and emotional comfort play a substantial role.

\section{Strategic Context}

As mentioned, mimicry of emotional expressions also depends on higher-order social cues in the perceiver's environment. However, the nature of this modulation is debated. Some argue that spontaneous mimicry is obligatory and difficult to inhibit (Brass et al. 2001; Cook et al. 2012). Others argue that even spontaneous responses to implicit emotional cues can be reshaped by context (Tamir et al. 2004). In order to address these questions, we ran another study with our android (Hofreeet al. 2018).

In this study, participants played multiple games with the android. In one block, participants were told that the android was their teammate (cooperative block), and in another block, that the android was their opponent (competitive block). Each game depended on chance, and its outcome was communicated to participants through the android's facial expressions (i.e., android was "smiling" when winning, and "frowning" when losing). Participants' facial expressions were measured using fEMG over the zygomaticus major and corrugator supercilii muscles.

Overall, participants spontaneously responded to the android in a way that expressed their own emotional reactions to the communicated valence of the game outcome-smiling to a win, frowning to a loss. Critically, participants displayed these expressions even when the android's expressions communicating the outcome were incongruent with their own. Specifically, during the competitive block, participants' smiled to the android's frown and frowned to the android's smile. Interestingly, in the competitive block participants' spontaneous facial reactions were fast and did not differ in timing or magnitude from their spontaneous reactions in the cooperative block (which were congruent with the android's).

In conclusion, this study suggests that basic, spontaneous mimicry reactions are shaped by social context. This has been previously discussed in research on reduced 
basic mimicry to outgroups (e.g., Bourgeois and Hess 2008; Likowski et al. 2008). Going beyond that, this android study suggests that rather than "suppressing" or "reducing" facial reactions, social context fundamentally reshapes them, such that they become spontaneous responses to the meaning of the observed expression (as in status and mimicry work by Carr et al. 2014).

Taking these studies on androids together, they suggest that spontaneous mimicry occurs in response to non-psychological agents but is also sensitive to basic physical similarity, including movement dynamics, and the social context in which those agents appear. This once again highlights the importance of considering mimicry within a broader notion of context, reflecting both the low-level cues and the inherent "intelligence" of the underlying process.

\section{Spontaneous Mimicry and Atypical Social Behavior}

So far, we have surveyed a variety of ways in which spontaneous mimicry functions in typical social cognition. We have argued that a full understanding of this phenomenon requires consideration of sensorimotor, cognitive, and emotional processes and how they are shaped by goals and a larger social context. In this section, we will argue that our analysis can be informed by (and hopefully inform) atypical social cognition. Here, we will consider (i) autism-a condition characterized by atypical social and emotional functioning and (ii) loneliness-perceived social isolation. We will also offer some speculation as to how differences in reward sensitivity, oxytocin levels, alexithymia, and interoception may play a role in these conditions. Our goal in this analysis is again to show that full understanding of mimicry requires appreciating that contextual variables operate on multiple levels.

\section{Autism}

Autism Spectrum Conditions (ASC) represent a constellation of symptoms characterized by atypicalities in three general areas: (i) social interaction, such as atypical social interest and behavior, (ii) communicative skills, including pragmatic language and gestures, and (iii) behavioral atypicalities, as with the presence of restricted or stereotyped patterns of behaviors, interests, and activities. Critically, autism is a multifaceted disorder. There is no widely-accepted biological cause of autism, and there is no single causal explanation, at any level, that explains all aspects of the syndrome. Further, the behavioral manifestations of this disorder vary in severity (e.g., low and high-functioning ASCs) and heterogeneity of the cognitive profile (e.g., language, intelligence, emotion, etc.). Unfortunately, different profiles of ASCs are often lumped together when reporting findings, sometimes obscuring the information about the level and domain of functioning for which the findings are relevant. Given that most research studies are conducted on high-functioning ASC individuals, they are most informative about this sub-group. Thus, it is worth keeping in mind that research across the spectrum of symptomology may reveal differential, idiosyncratic relationships amongst variables and outcomes. However, there do appear to be some consistent underlying mechanisms contributing to social challenges in ASC, such as spontaneous mimicry and reward processing. 


\section{Reduction and Delay in Spontaneous Mimicry of Facial Expressions}

There is a lively debate on ASC deficits in the domain of mimicry of gestures and faces. After all, ASC individuals can show hyperimitation (Spengler et al. 2010) and usually perform well on tests of deliberate mimicry (Rogers and Williams 2006). However, there is reasonable evidence for some specific impairments of spontaneous mimicry. For example, in one study, ASC individuals showed reduced activity in brain areas associated with preparation of motor actions when simply viewing videos of a person executing simple movements (Oberman et al. 2005). Several studies showed that, in contrast to typical participants, autistic individuals do not spontaneously mimic facial expressions when they "just watch" them, without any prompts to recognize or otherwise react to the expressions (Beall et al. 2008; McIntosh et al. 2006; Stel et al. 2008). Furthermore, even when autistic individuals explicitly focus on recognizing expressions, facial mimicry appears but is delayed (Oberman et al. 2009).

Note that these and related findings may reflect basic differences in attention to social stimuli as well as motivation and expertise (Southgate and de Hamilton 2008; Wang and de Hamilton 2012). When such factors are tightly controlled, it is possible to create conditions where ASC individuals can demonstrate intact spontaneous mimicry of hand actions (Bird et al. 2007) and facial expressions (Press et al. 2010; also see Cook et al. 2014b).

Whatever their origin, ASC impairments in spontaneous mimicry do occur and they can matter. First, individuals could suffer direct and indirect social consequences from dysregulated mimicry production. For example, impaired mimicry may reduce emotional contagion and shared social affect (for recent evidence from typical individuals, see Olszanowski et al. 2019). Furthermore, not demonstrating the expected social mimicry in context could fuel negative perceptions of the ASC individual, as mimicry is one way that individuals establish affiliation (Kavanagh and Winkielman 2016). Second, we know that spontaneous mimicry can facilitate emotion recognition. Thus, mimicry deficits may hinder recognition of facial expressions, at least under some conditions, making social interaction difficult. One clue for this comes from a study where ASC individuals performed selectively worse on a task involving recognition of rapidly presented expressions (Clark et al. 2008). Interestingly, there were no group differences when expressions were presented for longer durations. This could reflect ASC participants using non-embodied, rule-based, descriptive strategies for standard emotion recognition tasks (Rutherford and McIntosh 2007; Nuske et al. 2014; Winkielman et al. 2009). Finally, the idea that impairments in facial mimicry contribute to poor emotion recognition has also been highlighted in another atypical, neurodegenerative disorder associated with social difficulties-Parkinson's disease (Argaud et al. 2018). This suggests that relationships between spontaneous mimicry and other social-emotional processes are impactful across spectrums of (a)typical conditions, and worthy of examination into underlying mechanisms, with implications for improving human (social) life.

\section{Individual Differences: Oxytocin and Reward Processing}

Recent research suggests that neurobiological differences related to emotion processing are connected with atypical mimicry in ASC. Some of this research is based on the idea that dysregulation of the oxytocin (OT) system may play a role in ASC (Alvares et al. 2017). After all, OT is a neuropeptide associated with, among other physiological processes, 
attachment, sexuality, and facilitation of social responding (Carter 2014). Importantly, the mechanisms by which mimicry can influence social behavior are very complex and some effects in human studies that seem to reflect selective central OT mechanisms actually work via general peripheral causes (Churchland and Winkielman 2012) or are not very robust (Nave et al. 2015). Having said this, there is some evidence that variable levels of OT, along with other endocrine mechanisms, are involved in the neurocircuitry underlying mimicry (Kraaijenvanger et al. 2017). In accordance with its role in social processes, intranasal administration of OT in typical participants was shown to impact processes associated with facial emotion-multiple studies have reported improvement in emotion recognition (for a review, see Bakermans-Kranenburg and Van Ijzendoorn 2013), and another one demonstrated increased spontaneous facial mimicry (in males) as a likely mediating mechanism for such improved recognition (Korb et al. 2016). One potential explanatory mechanism for this is that OT increases the salience of social stimuli (Shamay-Tsoory and Abu-Akel 2016).

There is also evidence linking reduced spontaneous mimicry to atypical processing of social reward (Chevallier et al. 2012; Dichteret al. 2012; Kohls et al. 2012; Scott-Van Zeeland et al. 2010). This idea was tested in a psychophysiological study, which found that spontaneous facial mimicry was modulated by the reward value of different facial identities (Sims et al. 2012). In this experiment, neutral faces were first conditioned with high and low rewards using an evaluative conditioning task, instantiated through a card game. In the test phase, participants saw happy and angry expressions made by these same faces, while fEMG was recorded from congruent muscles to measure spontaneous facial mimicry. More rewarding faces were associated with greater spontaneous mimicry of joy, compared to less rewarding faces. Crucially, this reward-dependent modulation of spontaneous smile mimicry was inversely related to autistic traits (i.e., individuals high in autistic traits showed little facilitation of spontaneous mimicry for high versus low rewarding faces). An extension of these findings, using the same evaluative conditioning paradigm, showed that autistic traits did not moderate the extent of (implicit) conditioning (as measured by an implicit association test), but did reduce subsequent prosocial behavior toward the conditioned high-reward identities (Panasiti et al. 2016). This is especially interesting in the context of previous reports linking mimicry and prosocial behavior (van Baaren et al. 2004).

The relationship between autistic traits and reward-dependent modulation of mimicry was further demonstrated using hand stimuli in a task developed by Heyes and colleagues (e.g., Press et al. 2005). In this task, two human hand silhouettes were conditioned with high and low rewards using an implicit conditioning task as described above. In the test phase, participants were asked to make a pre-specified movement ('close' or 'open'), while a congruent or incongruent hand silhouette was presented simultaneously on the screen. The difference in reaction time to congruent versus incongruent stimuli was measured as a proxy metric of automatic imitation. Autistic traits were found to be inversely related to the reward-dependent modulation of automatic mimicry of human hands (Haffeyet al. 2013). Interestingly, this result was true only for human hands and not for robot hands, which were used as a control to test whether the reward-dependent modulation of automatic mimicry extended to non-human stimuli.

Generally, these studies suggest that autistic traits moderate the link between reward processing and spontaneous mimicry of social stimuli. This suggestion provides a potential theoretical bridge between studies that suggest a mirror system deficit in autism (Beall et al. 2008; Dapretto et al. 2006; McIntosh et al. 2006), and those that do not (Bird et al. 2007; Dinstein et al. 2010). Basically, the idea here is that the ASC individuals have 
atypical communication between the mirror system and the reward response to social stimuli. This view is in agreement with a framework suggesting atypical top-down modulation of mimicry in autism (Wang and de Hamilton 2012).

\section{Loneliness}

It is worth to note a similarity in reductions of reward-dependent mimicry in ASC to reduced expectations of social reward more generally. That is, if a person has less need for social actors, or has reasons to doubt their value, they should be less likely to mimic them. As discussed, individuals prefer people who provide them with social benefits and mimic them more. So, what happens when individuals expect negative social responses from others? One answer to this is the idea of hypervigilance for social threat, which is associated with loneliness-perceived social isolation (Cacioppo and Hawkley 2009). When an individual perceives their social connections as inadequate for an extended period (i.e., when chronically lonely), this maladaptive, implicit attentional bias may insidiously undermine social expectations and behavior. In our lab, we recently discovered a specific mimicry-related behavioral mechanism that may fuel the cycle of negative social expectations and inadequate social behavior inherent in loneliness (Arnold and Winkielman 2019). In our study, participants first completed the UCLA loneliness scale (Russell 1996) and then viewed emotional stimuli in three experimental sessions. In Session 1, participants passively viewed facial expression videos to assess spontaneous facial mimicry. In Session 2, participants viewed the same expressions again while instructed to deliberately mimic them. In Session 3, participants passively viewed emotionally-charged IAPS images to assess a more generic process of spontaneous smiling and frowning to valenced stimuli.

We found that loneliness was selectively associated with reduced spontaneous mimicry of smiles, with intact spontaneous mimicry of negative expressions (anger, fear, sadness). Importantly, there were no differences in deliberate mimicry of any expressions (Session 2), or in spontaneous smiling and frowning to emotional images (Session 3). The contrasting results between Sessions 1 and 3 suggest that loneliness is uniquely associated with less spontaneous mimicry of smiles directed at the participant. This is because spontaneous smiling to positively-valenced images (such as cute puppies and depictions of indirect positive social interactions) was not affected by loneliness.

Interestingly, explicit valence ratings of the smiles themselves did not differ as a function of loneliness. Implicit social reward perception may differ, however, and could mediate lower smile mimicry in loneliness. If so, expectation (or experience) of low social reward from others' positive social signals may undermine the very mechanisms that could facilitate positive emotional contagion and ultimately, social connection (Cacioppo and Hawkley 2009). This speculation is consistent with reports that loneliness is also associated with lower empathy (Beadle et al. 2012) and can be considered a type of learned social helplessness.

\section{Influences of Alexithymia and Interoception}

Finally, there are some recent suggestions that (a)typical social functioning is related to interoception - the sense of the physiological condition of the body (Arnold et al. 2019; Craig 2009). Interoceptive dysregulation has been found in ASC (see DuBois et al. 2016) with some intriguing links to oxytocin (Quattrocki and Friston 2014). However, since interoceptive dysregulation increases alexithymia — difficulty identifying one's own feelings and 
emotions-it has been argued that this underlies many of the social-emotional difficulties present in ASC (Mul et al. 2018; Shah et al. 2016). Specifically, it appears that lower interoception reduces emotional fidelity and thus could contribute to ASC through alexithymia. If one cannot accurately represent one's own feelings, it would be harder to simulate those of others (CODES; Winkielman et al. 2018). Indeed, dysregulated interoception has been theorized to impair aspects of empathy (Ondobaka et al. 2017; Palmer and Tsakiris 2018), including in ASC (Mul et al. 2018). We are not aware of data that directly link interoception and alexithymia to spontaneous mimicry, but this is an area of interesting inquiry.

\section{Summary and Conclusion}

When people interact socially, their success depends on many psychological and physiological mechanisms. Here we focused on one of them-spontaneous mimicry. We have shown how this response reflects a close link between perceptual, conceptual, motor and affective processes, and how it can play a causal role in emotion recognition, emotion understanding, contagion, and (potentially) empathy. We have highlighted some associative features of mimicry, its dependence on some perceptual features, but we have also highlighted its smart sensitivity to social context and its dependence of complex social variables such as social status or cooperative setting. Finally, we showed how investigating spontaneous mimicry can help us better understand atypical social behavior-notably how ASC and loneliness appear related to deficits in both the informative and affiliative properties of mimicry, resulting in impairments in basic emotion processing and unsatisfactory social interactions. In particular, it appears that atypical reward processing, interoception, and alexithymia may function as individual difference variables that (mis)shape mimicry responses, in part based on changes in one's own capacity to feel and label internal states. Without an accurate self-constructed "canvas" on which others emotions may make their mark, empathy, understanding, and social connection may be impaired. As such, some forms of social modulation of mimicry may be adaptive whereas other forms of modulation appear maladaptive. In conclusion, we suggest that spontaneous mimicry is one of the core processes that allows insight into the architecture of social cognition-where it goes right and where it sometimes goes astray.

Funding Funding was provided by Academic Senate Grant, University California, San Diego to Piotr Winkielman.

Open Access This article is distributed under the terms of the Creative Commons Attribution 4.0 International License (http://creativecommons.org/licenses/by/4.0/), which permits unrestricted use, distribution, and reproduction in any medium, provided you give appropriate credit to the original author(s) and the source, provide a link to the Creative Commons license, and indicate if changes were made.

\section{References}

Adolphs, R., Damasio, H., Tranel, D., Cooper, G., \& Damasio, A. R. (2000). A role for somatosensory cortices in the visual recognition of emotion as revealed by three-dimensional lesion mapping. The Journal of Neuroscience: The Official Journal of the Society for Neuroscience, 20(7), 2683-2690.

Alvares, G. A., Quintana, D. S., \& Whitehouse, A. J. (2017). Beyond the hype and hope: Critical considerations for intranasal oxytocin research in autism spectrum disorder. Autism Research, 10(1), 25-41. 
Anderson, J. R., Myowa-Yamakoshi, M., \& Matsuzawa, T. (2004). Contagious yawning in chimpanzees. Proceedings of the Royal Society of London, Series B: Biological Sciences, 271(Suppl_6), S468-S470.

Argaud, S., Vérin, M., Sauleau, P., \& Grandjean, D. (2018). Facial emotion recognition in Parkinson's disease: A review and new hypotheses. Movement Disorders, 33(4), 554-567.

Arnold, A., \& Winkielman, P. (2019). Smile (but only deliberately) though your heart is aching: Loneliness is associated with impaired spontaneous smile mimicry. (submitted for publication).

Arnold, A. J., Winkielman, P., \& Dobkins, K. (2019). Interoception and Social Connection. Frontiers in Psychology. https://doi.org/10.3389/fpsyg.2019.02589.

Bakermans-Kranenburg, M. J., \& van Ijzendoorn, M. H. (2013). Sniffing around oxytocin: review and meta-analyses of trials in healthy and clinical groups with implications for pharmacotherapy. Translational Psychiatry, 3(5), e258.

Barsalou, L. W. (1999). Perceptual symbol systems. Behavioral and Brain Sciences. https://doi. org/10.1017/s0140525x99002149.

Beadle, J. N., Keady, B., Brown, V., Tranel, D., \& Paradiso, S. (2012). Trait empathy as a predictor of individual differences in perceived loneliness. Psychological Reports, 110(1), 3-15. https://doi. org/10.2466/07.09.20.PR0.110.1.3-15.

Beall, P. M., Moody, E. J., McIntosh, D. N., Hepburn, S. L., \& Reed, C. L. (2008). Rapid facial reactions to emotional facial expressions in typically developing children and children with autism spectrum disorder. Journal of Experimental Child Psychology, 101, 206-223.

Bird, G., Leighton, J., Press, C., \& Heyes, C. (2007). Intact automatic imitation of human and robot actions in autism spectrum disorders. Proceedings of the Royal Society B: Biological Sciences, $274,3027$.

Blairy, S., Herrera, P., \& Hess, U. (1999). Mimicry and the judgment of emotional facial expressions. Journal of Nonverbal Behavior, 23(1), 5-37.

Bornemann, B., Winkielman, P., \& van der Meer, E. (2012). Can you feel what you do not see? Using internal feedback to detect briefly presented emotional stimuli. International Journal of Psychophysiology, 85(1), 116-124. https://doi.org/10.1016/j.ijpsycho.2011.04.007.

Bourgeois, P., \& Hess, U. (2008). The impact of social context on mimicry. Biological Psychology, 77, 343-352.

Brass, M., Bekkering, H., \& Prinz, W. (2001). Movement observation affects movement execution in a simple response task. Acta Psychologica, 106(1-2), 3-22.

Bulnes, L. C., Mariën, P., Vandekerckhove, M., \& Cleeremans, A. (2019). The effects of Botulinum toxin on the detection of gradual changes in facial emotion. Scientific Reports, 9(1), 11734. https:// doi.org/10.1038/s41598-019-48275-1.

Cacioppo, J. T., \& Hawkley, L. C. (2009). Perceived social isolation and cognition. Trends in Cognitive Sciences, 13(10), 447-454. https://doi.org/10.1016/j.tics.2009.06.005.

Calder, A., Keane, J., Cole, J., Campbell, R., \& Young, A. W. (2000). Facial expression recognition by people with Mobius syndrome. Cognitive Neuropsychology, 17(1-3), 73-87. https://doi. org/10.1080/026432900380490.

Calvo-Merino, B., Grèzes, J., Glaser, D. E., Passingham, R. E., \& Haggard, P. (2006). Seeing or doing? Influence of visual and motor familiarity in action observation. Current Biology, 16(19), 1905-1910.

Carr, E. W., Winkielman, P., \& Oveis, C. (2014). Transforming the mirror: Power fundamentally changes facial responding to emotional expressions. Journal of Experimental Psychology: General, 143, 997-1003.

Carter, C. S. (2014). Oxytocin pathways and the evolution of human behavior. Annual Review of Psychology, 65(1), 17-39. https://doi.org/10.1146/annurev-psych-010213-115110.

Chaminade, T., Franklin, D. W., Oztop, E., \& Cheng, G. (2005). Motor interference between humans and humanoid robots: Effect of biological and artificial motion. In The 4th international conference on development and learning, 2005. Proceedings (pp. 96-101).

Chevallier, C., Kohls, G., Troiani, V., Brodkin, E. S., \& Schultz, R. T. (2012). The social motivation theory of autism. Trends in Cognitive Sciences, 16(4), 231-239.

Churchland, P. S., \& Winkielman, P. (2012). Modulating social behavior with oxytocin: How does it work? What does it mean? Hormones and Behavior, 61, 392-399.

Clark, A. (1999). An embodied cognitive science? Trends in Cognitive Sciences, 3(9), 345-351. https:// doi.org/10.1016/S1364-6613(99)01361-3.

Clark, T. E., Winkielman, P., \& McIntosh, D. N. (2008). Autism and the extraction of emotion from briefly presented facial expressions: Stumbling at the first step of empathy. Emotion, 8(6), 803809. https://doi.org/10.1037/a0014124. 
Cook, R., Bird, G., Catmur, C., Press, C., \& Heyes, C. (2014a). Mirror neurons: From origin to function. Behavioral and Brain Sciences, 37, 177-241.

Cook, R., Bird, G., Lünser, G., Huck, S., \& Heyes, C. (2012). Automatic imitation in a strategic context: Players of rock-paper-scissors imitate opponents' gestures. Proceedings of the Royal Society B: Biological Sciences, 279(1729), 780-786.

Cook, R., Brewer, R., Shah, P., \& Bird, G. (2014b). Intact facial adaptation in autistic adults. Autism Research, 279, 780-786.

Cracco, E., Genschow, O., Radkova, I., \& Brass, M. (2018). Automatic imitation of pro- and antisocial gestures: Is implicit social behavior censored? Cognition, 170, 179-189. https://doi.org/10.1016/j.cogni tion.2017.09.019.

Craig, A. D. (2009). How do you feel-now? The anterior insula and human awareness. Nature Reviews Neuroscience, 10(1), 59-70. https://doi.org/10.1038/nrn2555.

Csibra, G., \& Gergely, G. (2009). Natural pedagogy. Trends in Cognitive Sciences, 13, 148-153.

Damasio, A. (1999). The feeling of what happens: Body and emotion in the making of consciousness. Mariner Books.

Dapretto, M., Davies, M., Pfeifer, J., Scott, A., Sigman, M., Bookheimer, S., et al. (2006). Understanding emotions in others: Mirror neuron dysfunction in children with autism spectrum disorders. Nature Neuroscience, 9, 28-30.

Davis, J. D., Winkielman, P., \& Coulson, S. (2017). Sensorimotor simulation and emotion processing: Impairing facial action increases semantic retrieval demands. Cognitive, Affective, and Behavioral Neuroscience, 17, 652-664.

Dichter, G. S., Richey, J. A., Rittenberg, A. M., Sabatino, A., \& Bodfish, J. W. (2012). Reward circuitry function in autism during face anticipation and outcomes. Journal of Autism and Developmental Disorders, 42, 147-160.

Dimberg, U. (1982). Facial reactions to facial expressions. Psychophysiology, 18, 643-647.

Dimberg, U., Thunberg, M., \& Elmehed, K. (2000). Unconscious facial reactions to emotional facial expressions. Psychological Science, 11, 86-89.

Dimberg, U., Thunberg, M., \& Grunedal, S. (2002). Facial reactions to emotional stimuli: Automatically controlled emotional responses. Cognition and Emotion, 16, 449-471. https://doi.org/10.1080/02699 930143000356.

Dinstein, I., Thomas, C., Humphreys, K., Minshew, N., Behrmann, M., \& Heeger, D. J. (2010). Normal movement selectivity in autism. Neuron, 66, 461-469.

DuBois, D., Ameis, S. H., Lai, M.-C., Casanova, M. F., \& Desarkar, P. (2016). Interoception in autism spectrum disorder: A review. International Journal of Developmental Neuroscience, 52, 104-111.

Duffy, K. A., \& Chartrand, T. L. (2015). Mimicry: Causes and consequences. Current Opinion in Behavioral Sciences, 3, 112-116.

Farmer, H., Carr, E. W., Svartdal, M., Winkielman, P., \& de Hamilton, A. F. (2016). Status and power do not modulate automatic imitation of intransitive hand movements. PLoS ONE, 11(4), e0151835. https:// doi.org/10.1371/journal.pone.0151835.

Fischer, A., \& Hess, U. (2017). Mimicking emotions. Current Opinion in Psychology, 17, 151-155.

Goldman, A. I., \& Sripada, C. S. (2005). Simulationist models of face-based emotion recognition. Cognition, 94, 193-213.

Haffey, A., Press, C., O’Connell, G., \& Chakrabarti, B. (2013). Autistic traits modulate mimicry of social but not nonsocial rewards: Autistic traits modulate mimicry of social rewards. Autism Research, 6 , 614-620.

Halberstadt, J., Winkielman, P., Niedenthal, P. M., \& Dalle, N. (2009). Emotional conception how embodied emotion concepts guide perception and facial action. Psychological Science, 20, 1254-1261.

Hess, U., \& Fischer, A. (2013). Emotional mimicry as social regulation. Personality and Social Psychology Review, 17(2), 142-157. https://doi.org/10.1177/1088868312472607.

Hofree, G., Ruvolo, P., Bartlett, M. S., \& Winkielman, P. (2014). Bridging the mechanical and the human mind: Spontaneous mimicry of a physically present android. PLOS ONE, 9(7), e99934.

Hofree, G., Ruvolo, P., Reinert, A., Bartlett, M. S., \& Winkielman, P. (2018). Behind the robot's smiles and frowns: In social context, people do not mirror androids' expressions but react to their informational value. Frontiers in Neurorobotics. https://doi.org/10.3389/fnbot.2018.00014.

Hofree, G., Urgen, B. A., Winkielman, P., \& Saygin, A. P. (2015). Observation and imitation of actions performed by humans, androids, and robots: An EMG study. Frontiers in Human Neuroscience. https:// doi.org/10.3389/fnhum.2015.00364.

Kavanagh, L. C., \& Winkielman, P. (2016). The functionality of spontaneous mimicry and its influences on affiliation: An implicit socialization account. Frontiers in Psychology. https://doi.org/10.3389/fpsyg .2016.00458. 
Keysers, C., Kaas, J. H., \& Gazzola, V. (2010). Somatosensation in social perception. Nature Reviews Neuroscience, 11, 417-428.

Kohls, G., Schulte-Rüther, M., Nehrkorn, B., Müller, K., Fink, G. R., Kamp-Becker, I., et al. (2012). Reward system dysfunction in autism spectrum disorders. Social Cognitive and Affective Neuroscience, 8(5), $565-572$.

Korb, S., Grandjean, D., \& Scherer, K. R. (2010). Timing and voluntary suppression of facial mimicry to smiling faces in a Go/NoGo task: An EMG study. Biological Psychology, 85(2), 347-349. https:// doi.org/10.1016/j.biopsycho.2010.07.012.

Korb, S., Malsert, J., Strathearn, L., Vuilleumier, P., \& Niedenthal, P. (2016). Sniff and mimic: Intranasal oxytocin increases facial mimicry in a sample of men. Hormones and Behavior, 84, 64-74. https://doi.org/10.1016/j.yhbeh.2016.06.003.

Korb, S., With, S., Niedenthal, P., Kaiser, S., \& Grandjean, D. (2014). The perception and mimicry of facial movements predict judgments of smile authenticity. PLOS ONE, 9(6), e99194. https://doi. org/10.1371/journal.pone.0099194.

Kraaijenvanger, E. J., Hofman, D., \& Bos, P. A. (2017). A neuroendocrine account of facial mimicry and its dynamic modulation. Neuroscience and Biobehavioral Reviews, 77, 98-106. https://doi. org/10.1016/j.neubiorev.2017.03.006.

Leighton, J., Bird, G., Orsini, C., \& Heyes, C. (2010). Social attitudes modulate automatic imitation. Journal of Experimental Social Psychology, 46(6), 905-910.

Likowski, K. U., Mühlberger, A., Seibt, B., Pauli, P., \& Weyers, P. (2008). Modulation of facial mimicry by attitudes. Journal of Experimental Social Psychology, 44(4), 1065-1072.

Lundqvist, L.-O., \& Dimberg, U. (1995). FaciaI expressions are contagious. Journal of Psychophysiology, 9, 203-211.

Maringer, M., Krumhuber, E. G., Fischer, A. H., \& Niedenthal, P. M. (2011). Beyond smile dynamics: Mimicry and beliefs in judgments of smiles. Emotion, 11, 181.

McIntosh, D. N., Reichmann Decker, A., Winkielman, P., \& Wilbarger, J. L. (2006). When the social mirror breaks: Deficits in automatic, but not voluntary, mimicry of emotional facial expressions in autism. Developmental Science, 9, 295-302.

Mesoudi, A. (2009). How cultural evolutionary theory can inform social psychology and vice versa. Psychological Review, 116, 929-952.

Molenberghs, P., Cunnington, R., \& Mattingley, J. B. (2012). Brain regions with mirror properties: A meta-analysis of 125 human fMRI studies. Neuroscience and Biobehavioral Reviews, 36, 341-349.

Moody, E. J., Mcintosh, D. N., Mann, L. J., \& Weisser, K. R. (2007). More than mere mimicry? The influence of emotion on rapid facial reactions to faces. Emotion, 7, 447-457.

Mul, C., Stagg, S. D., Herbelin, B., \& Aspell, J. E. (2018). The feeling of me feeling for you: Interoception, alexithymia and empathy in autism. Journal of Autism and Developmental Disorders, 48(9), 2953-2967.

Nadel, J., Simon, M., Canet, P., Soussignan, R., Blancard, P., Canamero, L., \& Gaussier, P. (2006). Human responses to an expressive robot. In Proceedings of the sixth international workshop on epigentic robotics (vol. 128, pp. 79-86). Lund University Cognitive Studies.

Nave, G., Camerer, C., \& McCullough, M. (2015). Does oxytocin increase trust in humans? A critical review of research. Perspectives on Psychological Science, 10(6), 772-789.

Neumann, R., Schulz, S., Lozo, L., \& Alpers, G. (2014). Automatic facial responses to near-threshold presented facial displays of emotion: Imitation or evaluation. Biological Psychology, 96, 144-149.

Niedenthal, P. M., Barsalou, L. W., Winkielman, P., Krauth-Gruber, S., \& Ric, F. (2005). Embodiment in attitudes, social perception, and emotion. Personality and Social Psychology Review, 9, 184-211.

Niedenthal, P. M., Brauer, M., Halberstadt, J. B., \& Innes-Ker, A. H. (2001). When did her smile drop? Contrast effects in the influence of emotional state on the detection of change in emotional expression. Cognition and Emotion, 15, 853-864.

Niedenthal, P. M., Winkielman, P., Mondillon, L., \& Vermeulen, N. (2009). Embodiment of emotional concepts: Evidence from EMG measures. Journal of Personality and Social Psychology, 96, 1120-1136.

Nuske, H., Vivanti, G., Hudry, K., \& Dissanayake, C. (2014). Pupillometry reveals reduced unconscious emotional reactivity in autism. Biological Psychology, 101, 24-35.

Oberman, L. M., Hubbard, E. M., McCleery, J. P., Altschuler, E. L., Ramachandran, V. S., \& Pineda, J. A. (2005). EEG evidence for mirror neuron dysfunction in autism spectrum disorders. Cognitive Brain Research, 24(2), 190-198.

Oberman, L. M., Winkielman, P., \& Ramachandran, V. S. (2007). Face to face: Blocking facial mimicry can selectively impair recognition of emotional expressions. Social Neuroscience, 2(3-4), 167-178. 
Oberman, L. M., Winkielman, P., \& Ramachandran, V. S. (2009). Slow echo: Facial EMG evidence for the delay of spontaneous, but not voluntary, emotional mimicry in children with autism spectrum disorders. Developmental Science, 12(4), 510-520.

Olszanowski, M., Wróbel, M., \& Hess, U. (2019). Mimicking and sharing emotions: A re-examination of the link between facial mimicry and emotional contagion. Cognition and Emotion. https://doi. org/10.1080/02699931.2019.1611543.

Ondobaka, S., Kilner, J., \& Friston, K. (2017). The role of interoceptive inference in theory of mind. Brain and Cognition, 112, 64-68. https://doi.org/10.1016/j.bandc.2015.08.002.

Palagi, E., Tamietto, M., Celeghin, A., Winkielman, P. \& Norscia, I. (under review). The neuroethology of spontaneous mimicry and emotional contagion in human and non-human animals. Neuroscience and Biobehavioral Reviews.

Palmer, C. E., \& Tsakiris, M. (2018). Going at the heart of social cognition: Is there a role for interoception in self-other distinction? Current Opinion in Psychology, 24, 21-26. https://doi.org/10.1016/j. copsyc.2018.04.008.

Panasiti, M. S., Puzzo, I., \& Chakrabarti, B. (2016). Autistic traits moderate the impact of reward learning on social behaviour: Reward, prosocial behaviour, and autistic traits. Autism Research, 9(4), 471-479. https://doi.org/10.1002/aur.1523.

Pitcher, D., Garrido, L., Walsh, V., \& Duchaine, B. C. (2008). Transcranial magnetic stimulation disrupts the perception and embodiment of facial expressions. Journal of Neuroscience, 28(36), 8929-8933. https://doi.org/10.1523/jneurosci.1450-08.2008.

Press, C., Bird, G., Flach, R., \& Heyes, C. (2005). Robotic movement elicits automatic imitation. Cognitive Brain Research, 25(3), 632-640.

Press, C., Richardson, D., \& Bird, G. (2010). Intact imitation of emotional facial actions in autism spectrum conditions. Neuropsychologia, 48(11), 3291-3297.

Quattrocki, E., \& Friston, K. (2014). Autism, oxytocin and interoception. Neuroscience and Biobehavioral Reviews, 47, 410-430.

Rives-Bogart, K., \& Matsumoto, D. (2010). Facial mimicry is not necessary to recognize emotion: Facial expression recognition by people with Moebius syndrome. Social Neuroscience, 5(2), 241-251.

Rogers, S. J., \& Williams, J. H. G. (2006). Imitation and the social mind: Autism and typical development. NY: Guilford Press.

Russell, D. W. (1996). UCLA Loneliness Scale (Version 3): Reliability, validity, and factor structure. Journal of Personality Assessment, 66(1), 20-40.

Rutherford, M. D., \& McIntosh, D. N. (2007). Rules versus prototype matching: Strategies of perception of emotional facial expressions in the autism spectrum. Journal of Autism and Developmental Disorders, 37(2), 187-196.

Rychlowska, M., Cañadas, E., Wood, A., Krumhuber, E. G., Fischer, A., \& Niedenthal, P. M. (2014). Blocking mimicry makes true and false smiles look the same. PLOS ONE, 9(3), e90876.

Schubert, T. W., \& Semin, G. R. (2009). Embodiment as a unifying perspective for psychology. European Journal of Social Psychology, 39(7), 1135-1141.

Scott-Van Zeeland, A. A., Dapretto, M., Ghahremani, D. G., Poldrack, R. A., \& Bookheimer, S. Y. (2010). Reward processing in autism. Autism Research: Official Journal of the International Society for Autism Research, 3, 53.

Seibt, B., Mühlberger, A., Likowski, K. U., \& Weyers, P. (2015). Facial mimicry in its social setting. Frontiers in Psychology. https://doi.org/10.3389/fpsyg.2015.01122.

Shah, P., Hall, R., Catmur, C., \& Bird, G. (2016). Alexithymia, not autism, is associated with impaired interoception. Cortex, 81, 215-220.

Shamay-Tsoory, S. G., \& Abu-Akel, A. (2016). The social salience hypothesis of oxytocin. Biological Psychiatry, 79(3), 194-202. https://doi.org/10.1016/j.biopsych.2015.07.020.

Sims, T. B., Van Reekum, C. M., Johnstone, T., \& Chakrabarti, B. (2012). How reward modulates mimicry: EMG evidence of greater facial mimicry of more rewarding happy faces. Psychophysiology, 49(7), 998-1004.

Sonnby-Borgstrom, M. (2016). Emotional mimicry: Underlying mechanisms and individual differences. In U. Hess \& A. Fischer (Eds.), Emotional mimicry in social context (pp. 125-161). Cambridge, UK: Cambridge University Press.

Southgate, V., \& de Hamilton, A. F. C. (2008). Unbroken mirrors: challenging a theory of autism. Trends in Cognitive Sciences, 12(6), 225-229.

Spengler, S., Bird, G., \& Brass, M. (2010). Hyperimitation of actions is related to reduced understanding of others' minds in autism spectrum conditions. Biological Psychiatry, 68(12), 1148-1155.

Stel, M., \& van Knippenberg, A. (2008). The role of facial mimicry in the recognition of affect. Psychological Science, 19(10), 984-985. 
Stel, M., van den Heuvel, C., \& Smeets, R. C. (2008). Facial feedback mechanisms in autistic spectrum disorders. Journal of Autism and Developmental Disorders, 38(7), 1250-1258.

Tamir, M., Robinson, M. D., Clore, G. L., Martin, L. L., \& Whitaker, D. J. (2004). Are we puppets on a string? The contextual meaning of unconscious expressive cues. Personality and Social Psychology Bulletin, 30, 237-249.

Tiedens, L. Z., \& Fragale, A. R. (2003). Power moves: Complementarity in dominant and submissive nonverbal behavior. Journal of Personality and Social Psychology, 84, 558-568.

Tomasello, M., Kruger, A. C., \& Ratner, H. H. (1993). Cultural learning. Behavioral and Brain Sciences, 16(03), 495-511.

van Baaren, R. B., Holland, R. W., Kawakami, K., \& van Knippenberg, A. (2004). Mimicry and prosocial behavior. Psychological Science, 15(1), 71-74.

Wang, Y., \& de Hamilton, A. F. C. (2012). Social top-down response modulation (STORM): A model of the control of mimicry in social interaction. Frontiers in Human Neuroscience, 6(153), 1-10.

Waytz, A., Cacioppo, J., \& Epley, N. (2010). Who sees human? The stability and importance of individual differences in anthropomorphism. Perspectives on Psychological Science, 5, 219-232.

Weyers, P., Mühlberger, A., Hefele, C., \& Pauli, P. (2006). Electromyographic responses to static and dynamic avatar emotional facial expressions. Psychophysiology, 43, 450-453.

Weyers, P., Mühlberger, A., Kund, A., Hess, U., \& Pauli, P. (2009). Modulation of facial reactions to avatar emotional faces by nonconscious competition priming. Psychophysiology, 46, 328-335.

Wilson, M. (2002). Six views of embodied cognition. Psychonomic Bulletin \& Review, 9(4), 625-636. https ://doi.org/10.3758/bf03196322.

Winkielman, P., Coulson, S., \& Niedenthal, P. (2018). Dynamic grounding of emotion concepts. Philosophical Transactions of the Royal Society B: Biological Sciences, 373(1752), 20170127.

Winkielman, P., McIntosh, D. N., \& Oberman, L. (2009). Embodied and disembodied emotion processing: Learning from and about typical and autistic individuals. Emotion Review, 2, 178-190.

Winkielman, P., Niedenthal, P., Wielgosz, J., Eelen, J., \& Kavanagh, L. C. (2015). Embodiment of cognition and emotion. In M. Mikulincer, P. R. Shaver, E. Borgida, \& J. A. Bargh (Eds.), APA handbook of personality and social psychology (Vol. 1, pp. 151-175)., Attitudes and social cognition Washington, DC: APA.

Wood, A., Rychlowska, M., Korb, S., \& Niedenthal, P. (2016). Fashioning the face: Sensorimotor simulation contributes to facial expression recognition. Trends in Cognitive Sciences, 20(3), 227-240. https ://doi.org/10.1016/j.tics.2015.12.010.

Wu, T., Butko, N. J., Ruvulo, P., Bartlett, M. S., \& Movellan, J. R. (2009). Learning to make facial expressions. In Proceedings of the 2009 IEEE 8th international conference on development and learning (pp. 1-6). IEEE.

Publisher's Note Springer Nature remains neutral with regard to jurisdictional claims in published maps and institutional affiliations. 